\section{Brustkrebs: etwas Alkohol vorteilhaft}

\author{
Welchen Einfluss hat ein geringer bis moderater Alkoholkonsum auf das \\ Überleben nach Brustkrebs? Eine Kohortenstudie mit etwa 23.000 erkrankten \\ Frauen liefert einige überraschende Hinweise.
}

\begin{abstract}
Die ie Frauen wurden einerseits zu Beginn retrospektiv über ihren Alkoholkonsum befragt, zum anderen liegen prospektive Daten von knapp 4.900 Frauen zum Alkoholkonsum nach der Tumordiagnose vor. Zu Beginn der Studie waren $19 \%$ der Frauen abstinent. Von diesen waren im Schnitt elf Jahre später $16,5 \%$ an Brustkrebs gestorben. Von den Frauen, die 10-20 g Alkohol pro Woche tranken - das war die Hälfte starben im selben Zeitraum nur 15,4\% an dem Tumor. Am niedrigsten war die Sterberate bei Frauen mit einem Konsum von 30-60 g Alkohol pro Woche mit 13,6\%, danach stieg sie wieder leicht an, lag aber selbst bei Frauen mit über $100 \mathrm{~g}$ pro Woche noch bei $15,5 \%$ und damit niedriger als bei den Abstinenzlern.
\end{abstract}

Wurden verschiedene Faktoren wie Alter, Tumorstadium oder BrustkrebsPrädispositionen berücksichtigt, so war die brustkrebsbedingte Sterberate bei Frauen mit 30-60 g Alkohol pro Woche um $15 \%$ geringer als bei Abstinenzlern. Noch deutlicher war der Zusammenhang bei kardiovaskulär bedingten Sterbefällen. Diese waren in der Gruppe mit 70-90 g Alkohol um 25\% niedriger als bei den Frauen ohne jeglichen Alkoholkonsum. Außerdem war auch die Gesamtsterberate in der Gruppe mit 3060 g Alkohol pro Woche um $20 \%$ niedriger als bei den Abstinenzlern. Doch bei hohem Alkoholkonsum verschwand der Nutzen auch wieder: Frauen mit mehr als $100 \mathrm{~g}$ pro Woche hatten keinen Vorteil bei der Gesamtüberlebensrate.
Als nächstes nahmen die Autoren die Daten der 4.900 Frauen unter die Lupe, von denen auch Angaben zu Trinkgewohnheiten nach der Krebsdiagnose vorlagen. Insgesamt ergab sich ein ähnliches Bild wie in der Gesamtkohorte: Auch hier wurde die Brustkrebsmortalität, die kardiovaskuläre Mortalität und die Gesamtsterberate durch mäßigen Alkoholkonsum leicht, wenn auch nicht signifikant reduziert.

Fazit: Trinken Frauen vor oder nach der Brustkrebsdiagnose moderat Alkohol, hat das keine negativen Folgen - im Gegenteil: Einen frühen Tod durch Brustkrebs und vor allem Gefäßerkrankungen müssen sie weniger fürchten. Allerdings, so die Autoren, könnte die Situation bei Östrogenrezeptor-positiven und -negativen Tumoren anders sein. Thomas Müller

Newcomb PA et al. Alcohol consumption before and after breast cancer diagnosis: Associations with survival from breast cancer, cardiovascular disease, and other causes. J Clin Oncol 2013; Apr 8 [Epub ahead of print].

\section{Prädiktoren nach neoadjuvanter Chemotherapie}

\begin{abstract}
Während es für Patientinnen mit frühem Mammakarzinom, die primär operiert werden, belastbare Prädiktoren gibt, ist in der neoadjuvanten Situation nicht klar, wer von einer postoperativen Bestrahlung profitiert und wer nicht. Erstmals liegen jetzt prospektive Daten zu dieser Frage vor.
\end{abstract}

$\mathrm{D}$ ie Daten zu Prädiktoren eines lokoregionären Rezidivs (LRR) als erstes Ereignis nach der Behandlung eines operablen Mammakarzinoms basieren auf zwei neoadjuvanten Studien des NSABP. (National Surgical Adjuvant Breast and Bowel Project). Die neoadjuvante Chemotherapie (NC) bestand dabei entweder aus Doxorubicin plus Cyclophosphamid alleine (AC) oder mit nachfolgendem neoadjuvantem und adjuvantem Docetaxel. Wurde nur eine Lumpektomie durchgeführt, erfolgte regelhaft eine adjuvante Strahlentherapie, nicht jedoch nach Mastektomie.

3.088 Patientinnen wurden zehn Jahre nachbeobachtet. Die kumulative 10-Jahres-Inzidenz lag bei MastektomiePatientinnen bei $12,3 \%$ (8,9\% lokal; $3,4 \%$ regionär) und bei Patientinnen nach Lumpektomie und adjuvanter Radiotherapie bei $10,3 \%$ (8,1 \% lokal; $2,2 \%$ regionär). Die multivariate Analyse ergab als unabhängige Prädiktoren für ein LRR nach Lumpektomie das Alter, den klinisch ermittelten Lymphknotenstatus vor NC und den pathologischen Nodalstatus nach bzw. Tumoransprechen auf die NC. Bei den Patientinnen mit Mastektomie identifizierten die Untersucher als unabhängige Prädiktoren für ein LRR die klinische Tumorgröße vor der NC und wiederum den pathologischen Nodalstatus/Tumoransprechen nach NC. Aufgrund dieser Prädiktoren ließen sich Gruppen mit niedrigem, mittlerem und hohem Risiko für ein LRR identifizieren und Nomogramme erstellen.

Ein Kommentator weist darauf hin, dass eine Schwäche der Studie in der al- lein klinischen Beurteilung des Tumors und des Lymphknotenstatus vor NC liegt. Auch ist das paradoxe Ergebnis, dass Patientinnen ohne Lymphknotenbefall zu Beginn und mit komplettem Ansprechen nach der NC mit einem 10-Jahres-Risiko für ein LRR von 6,26,5\% eine schlechtere Prognose haben als Patientinnen mit klinisch positivem Nodalstatus, möglicherweise ein Hinweis auf weitere Prädiktoren.

Fazit: Bei Patientinnen mit operablem Mammakarzinom, die eine NC erhalten, lassen Faktoren wie Alter, klinische Tumorcharakteristika und Ansprechen auf NC eine Einschätzung des Risikos für ein LRR zu und stützen damit auch die Entscheidung für oder gegen adjuvante Strahlentherapie. Friederike Klein

Mamounas EP et al. Predictors of locoregional recurrence after neoadjuvant chemotherapy: Results from combined analysis of National Surgical Adjuvant Breast and Bowel Project B-18 and B-27. J Clin Oncol. 2012;30(32):3960-6. Kommentar von Smith BD. Using chemotherapy response to personalize choices regarding locoregional Therapy: A new era in breast cancer treatment? Ibidem 3913-4. 